\title{
Crystal structure of human apolipoprotein A-I: Insights into its protective effect against cardiovascular diseases
}

\author{
A. Abdul Ajees*, G. M. Anantharamaiah ${ }^{\dagger}$, Vinod K. Mishra $^{\dagger}$, M. Mahmood Hussain ${ }^{\ddagger}$, and H. M. Krishna Murthy*§ \\ *Center for Biophysical Sciences and Engineering and ${ }^{\dagger}$ Atherosclerosis Research Unit and Departments of Medicine, Biochemistry, and Molecular Genetics, \\ University of Alabama, 1530 3rd Avenue South, Birmingham, AL 35294; and ₹Departments of Anatomy, Cell Biology, and Pediatrics, State University of \\ New York Downstate Medical Center, Brooklyn, NY 11203
}

Edited by John A. Glomset, University of Washington, Seattle, WA, and approved December 20, 2005 (received for review August 9, 2005)

\begin{abstract}
Despite three decades of extensive studies on human apolipoprotein A-I (apoA-I), the major protein component in high-density lipoproteins, the molecular basis for its antiatherogenic function is elusive, in part because of lack of a structure of the full-length protein. We describe here the crystal structure of lipid-free apoA-I at $2.4 \AA$. The structure shows that apoA-I is comprised of an $\mathrm{N}$-terminal four-helix bundle and two C-terminal helices. The $\mathrm{N}$-terminal domain plays a prominent role in maintaining its lipid-free conformation, indicating that mutants with truncations in this region form inadequate models for explaining functional properties of apoA-I. A model for transformation of the lipid-free conformation to the high-density lipoprotein-bound form follows from an analysis of solvent-accessible hydrophobic patches on the surface of the structure and their proximity to the hydrophobic core of the four-helix bundle. The crystal structure of human apoA-I displays a hitherto-unobserved array of positively and negatively charged areas on the surface. Positioning of the charged surface patches relative to hydrophobic regions near the $C$ terminus of the protein offers insights into its interaction with cell-surface components of the reverse cholesterol transport pathway and antiatherogenic properties of this protein. This structure provides a much-needed structural template for exploration of molecular mechanisms by which human apoA-I ameliorates atherosclerosis and inflammatory diseases.
\end{abstract}

helical structure | high-density lipoproteins | x-ray crystal structure atherosclerosis | lipid-free structure

$T^{1}$ he importance of human apolipoprotein A-I (apoA-I) in protection against cardiovascular disease is underscored by recent studies demonstrating that infusion of recombinant highdensity lipoproteins (HDL) consisting of apoA- $\mathrm{I}_{\text {Milano }}$ and palmitoyloleoylphosphatidylcholine complexes in humans shows regression of preexisting atherosclerotic plaques (1). More than 30 years of sustained research (2) has gone into understanding the mechanism(s) of protection that apoA-I provides against heart disease. The protective action of apoA-I and HDL is attributed to their central role in reverse cholesterol transport (RCT); a process in which apoA-I acts as an acceptor for sequential transfers of phospholipids and free cholesterol (FC) from peripheral tissues and transports cholesterol to the liver and other tissues for excretion and steroidogenesis $(3,4)$. Modulation of the RCT pathway presents significant potential for treatment of heart disease (5). ApoA-I in both lipid-free and lipid-bound forms plays critical roles in each of the three steps in RCT: $(i)$ transfer of FC to acceptors mediated by ATP-binding cassette transporter A1 (ABCA1); (ii) esterification of $\mathrm{FC}$ to form cholesteryl esters (CE) by HDL-associated enzyme lecithin cholesterol acyl transferase (LCAT); and (iii) delivery of CE to the liver mediated by scavenger receptor class B type I (SR-B1). ApoA-I interacts with ABCA1 and accepts FC in the first step, acts as a cofactor for LCAT in the second, and mediates interaction of HDL with SR-B1 in the third (6-8). Additionally,
HDL and apoA-I play a significant role in cholesterol absorption (9), modulate endothelial function, inhibit platelet-activating factor synthesis, stimulate prostacyclin synthesis, bind to bacterial lipopolysaccharide, and inhibit low-density lipoproteininduced monocyte chemotaxis (10).

ApoA-I exists in multiple conformations, with metabolic interconversion among lipid-free/poor, partially lipidated, and fully lipidated states, triggered by ambient lipid concentration, occurring in vivo (reviewed in refs. 11-15). Physiologically, a lipid-free form of apoA-I exists in a thermodynamically labile state but is rapidly lipidated, resulting in nearly all of the apoA-I in vivo being lipid-bound $(11-13,16)$. However, evidence is accumulating on lipid-free/poor apoA-I being the primary acceptor of FC from peripheral cells (16) and as the substrate for myeloperoxidase-mediated chlorination, which impairs ABCA1mediated cholesterol efflux (17). Despite extensive studies because of its importance in combating cardiovascular diseases, a three-dimensional structure of full-length apoA-I has been unavailable. We report here the crystal structure of human apoA-I at 2.4- $\AA$ resolution. The structure consists of two helical domains; a four-helix antiparallel bundle formed by the $\mathrm{N}$ terminal three-quarters of apoA-I and a two-helix bundle adopted by the C-terminal quarter of the molecule. It also reveals the prominent role played by the N-terminal domain of apoA-I in stabilizing its lipid-free conformation. A lipidaccessible surface hydrophobic patch at the base of the four-helix bundle permits us to suggest a simple model for its conversion to an open HDL-bound conformation, a key functional property of apoA-I. In addition, examination of electrostatic features of the structure provides insights into interactions of apoA-I with ABCA1 and SR-B1. Finally, our results will form the basis for structure-based mutational and other experiments to understand the molecular basis of the central role played by apoA-I in protection against cardiovascular disease.

\section{Results and Discussion}

Lipid-Free ApoA-I Is Largely Helical. The $\mathrm{C}^{\alpha}$ atoms of the three apoA-I molecules in the asymmetric unit can be superimposed with rms deviations of $0.74,0.53$, and $0.84 \AA$, respectively, for molecule pairs. Each apoA-I molecule is organized into a pair of antiparallel helical bundles, a four-helix bundle at the $\mathrm{N}$ terminus and a two-helix bundle at the $\mathrm{C}$ terminus (Fig. 1). The three

Conflict of interest statement: No conflicts declared.

This paper was submitted directly (Track II) to the PNAS office.

Freely available online through the PNAS open access option.

Abbreviations: ApoA-I, apolipoprotein A-I; HDL, high-density lipoproteins; $\mathrm{Cr}$-acac3, $\mathrm{Cr}$ (III)tris-acetylacetonate; ABCA1, ATP-binding cassette transporter A1; LCAT, lecithin cholesterol acyl transferase; SR-B1, scavenger receptor class B type I; FC, free cholesterol.

Data deposition: The atomic coordinates and structure factors have been deposited in the Protein Data Bank, www.pdb.org (PDB ID code 2A01).

§To whom correspondence should be addressed. E-mail: murthy@cbse.uab.edu.

C 2006 by The National Academy of Sciences of the USA 


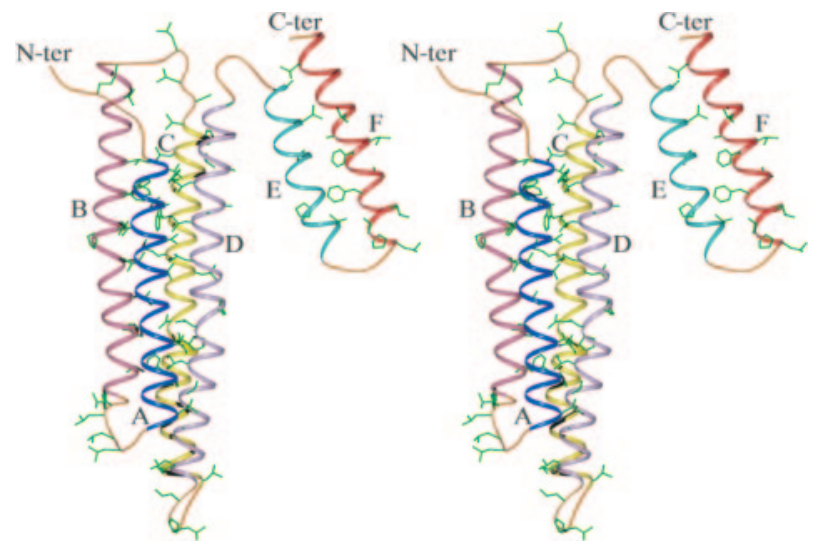

Fig. 1. Overall stereoview of the structure. The six helices in the structure are rendered as $C^{\alpha}$ worms, colored blue (A), pink (B), yellow (C), lavender (D), cyan $(E)$, and red (F) and labeled. Loops are colored gold. Hydrophobic residues are shown as green sticks.

molecules of apoA-I in the asymmetric unit are clustered around the threefold axis of a $\mathrm{D}_{3}$ symmetric $\mathrm{Cr}$ (III)-tris-acetylacetonate (Cr-acac3) moiety and are related by approximate threefold symmetry, with rotations of $129^{\circ}, 117^{\circ}$, and $114^{\circ}$. van der Waals interactions made by a hydrophobic patch formed by Leu-218, -219 , and -222 of each apoA-I molecule and this Cr-acac3 moiety stabilize the trimer (see Fig. 6, which is published as supporting information on the PNAS web site). Experimental electron density around this region is shown in Fig. 7, which is published as supporting information on the PNAS web site. A notable feature of the structure is the presence of longer helices than anticipated from sequence analysis and the absence of strict proline punctuation observed in $\Delta 1-43$ apoA-I (18). Like two prolines in L. migratoria apolipophorin III (19), and several in apoA-II (20), Pro-66, -121, and -165 occur in the middle of helices. Helices A-D of the four-helix bundle are shown in Fig. 1 , and the residue correspondence between sequence based helices (15) and positions of prolines are presented in Fig. 8, which is published as supporting information on the PNAS web site. Pairs of $\mathrm{Cr}$-acac3 molecules bridge the two helical domains of each apoA-I through interaction with residues Arg-171, Gln-172, Ala-176, Asn-184, Arg-188, His-193, Lys-195, His-199, Lys-206, Ala-210, and Tyr-236 (see Fig. 9, which is published as supporting information on the PNAS web site). Contacts between protein and $\mathrm{Cr}$-acac3 are critical to the maintenance of a compact configuration of the two-domain structure and the trimer, explaining the requirement of $\mathrm{Cr}$-acac3 for growth of crystals. The helices are connected by mostly type IV $\beta$ turns, although some type I $\beta$ and $\gamma$ turns also occur. Three residues (90-92) in the bc loop of one monomer of apoA-I form one turn of a $3_{10}$ helix. The first nine $\mathrm{N}$-terminal residues form an inverse $\gamma$ turn while Gln-243 at the C terminus is in a nonhelical conformation. The N-terminal loop is stabilized through crystal contacts, especially of Asp-1, Glu-2, and Pro-3 with crystallographically related Gln-84, Lys-140, Leu-141, and Ser-142. Surface hydrophobic patches on apolipoproteins, including apoA-I, have been shown to cause oligomerization in solution (21). However, except in case of apoA-II (20) and truncated apoA-I (18), such oligomerization has not been observed in apolipoprotein crystal structures (19), including in this structure (see Fig. 10 , which is published as supporting information on the PNAS web site). The four-helix bundle is stabilized by the shielding from solvent of $6,582 \AA^{2}$ of accessible surface area of residues in the interface. The hydrophobic core of the bundle is formed by side chains from all four helices in roughly equal proportion. In particular, the N-terminal domain (residues 1-43, helix A), forms an integral part of the bundle, and its deletion likely results in the open structure of the $\Delta 1-43$ mutant. Importance of this domain for structural integrity is supported by mutations in this region that lead to misfolded apoA-I variants found in amyloid deposits (22). Based on various experimental observations, subsets of residues forming helices 3-6 have been suggested to form a hinged domain, important for formation of a stable A-I belt in discoidal complexes and for subspeciation of HDL (23). In our structure, the average B factor in this region is similar to other helical regions, suggesting equivalent mobility. Residues 85-96 and 138-145 form the ab and bc loops (Fig. 1), providing mobile regions at either end of helix B (helices 4 and 5), which could potentially act as hinges. Antibody binding studies have identified the 4-5 helix pair as the hinged mobile region (24).

Measured helical content for lipid-free apoA-I in solution varies from $50 \%$ (25) to $>74 \%$ (26), depending on the aggregation state. In our structure, $\approx 82 \%$ of residues are in helices A-E (Figs. 1 and 8). The high concentration of the protein used in crystallization experiments is likely to induce aggregation, which generates, around each copy of apoA-I, a more hydrophobic environment than in dilute solution, resulting in a helical content closer to the lipid-bound state. Unlike in the crystal structure of apoA-II (20), hydrophobic association does not persist in this structure. Nevertheless, crystal packing presumably prevents relaxation to a helical content seen in dilute solution. ApoA-I has long been considered to be a "molten globule" in solution, adopting an ensemble of conformations, contributing to difficulty of crystallization (11). The low concentration of guanidine hydrochloride $(1.2 \mathrm{M})$ for $50 \%$ unfolding $\left(c_{1 / 2}\right)$, emphasizes this characteristic (11). However, recent crosslinking data have implied a well defined four-helix bundle structure for the N-terminal domain in lipid-free solution (27). In addition, insect lipoproteins, apoLp-III from Locusta migratoria $\left[c_{1 / 2}=0.6 \mathrm{M}(28)\right]$ and Manduca sexta $\left[c_{1 / 2}=0.4 \mathrm{M}(29)\right]$ with even smaller estimated thermodynamic stabilities, form stable helical bundles in their crystal structures. ApoA-I helices unfold independently on thermal denaturation (30), although equivalent data are not available for the insect proteins. Together, these results suggest conformation adopted by apoA-I in this structure is one of the conformations adopted by the protein in solution.

Comparison with Other Apolipoproteins. The structure of the fulllength protein measures $88 \times 50 \times 27 \AA$ at its widest point, with the four-helix bundle accounting for $88 \times 25 \times 27 \AA$. Two of the dimensions of the four-helix bundle are similar to those of the N-terminal domain of apoE $(65 \times 22 \times 24 \AA)(31)$, although the longer apoA-I helices make its structure taller. Superimposition of the 118 common $\alpha$ carbon atoms of apoA-I and apoE four-helix bundles yields a rms deviation of $3.2 \AA$. Most of the difference arises from dissimilarities in the loops between the two proteins and disparity in inclination of helix axes. In particular, helices 1 and 2 in apoE are connected by an 11-residue helix, and the loops between other helices have different lengths (Fig. $2 A$ ). Each copy of apoA-I in the asymmetric unit is in part stabilized by an average of 133 hydrogen bonds that are $50 \%$ or more buried in the four-helix bundle, whereas the apoE bundle has 121 . Because of the longer helices in apoA-I (Fig. $2 A$ ), there are 157 residues in its bundle, contrasted with 118 in apoE. Thus, the apoA-I bundle buries an average of 0.71 hydrogen bond per residue compared with 1.03 for apoE. Similarly, each residue in the apoA-I bundle buries, on average, $42 \AA^{2}$ of surface area vs. $54 \AA^{2}$ in apoE. Both these measures indicate that the apoA-I four-helix bundle is less stable than that in apoE, in agreement with its smaller measured thermodynamic stability (11).

The crystal structure of apoA-I described here differs markedly from the crystal structure of $\Delta 1-43$ apoA-I, which is elongated $(125 \times 80 \times 40 \AA)$ and open (18), and similar in 

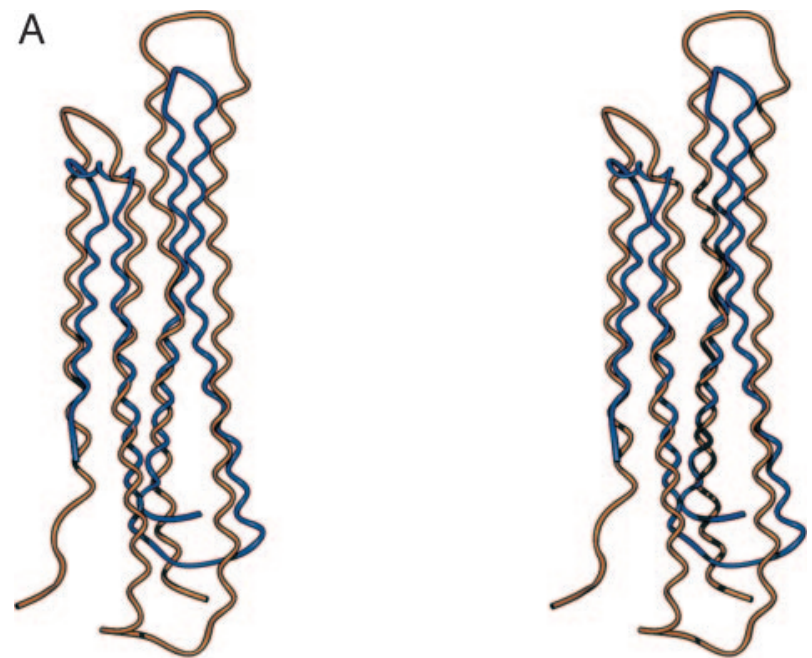

B

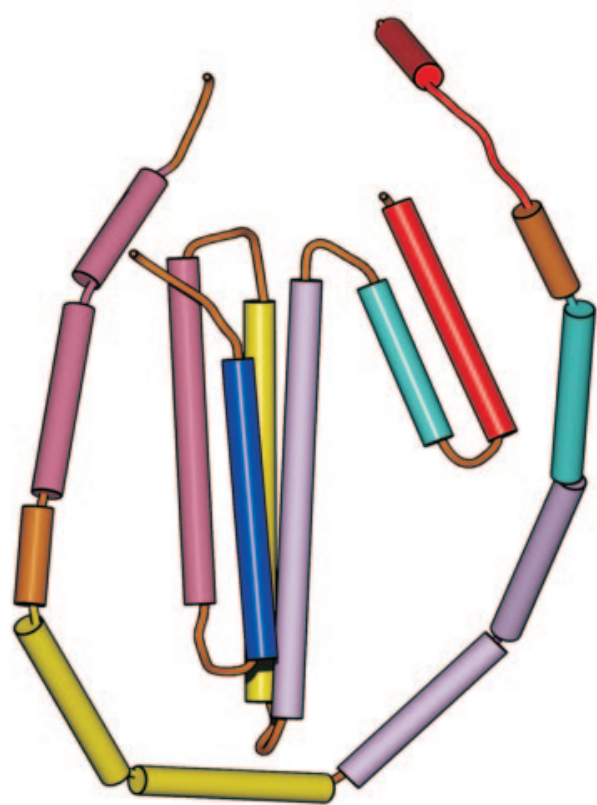

Fig. 2. Comparison with other apolipoproteins. (A) Stereoview of superimposition of the $\mathrm{C}^{\alpha}$ worm of lipid-free apoA-I over that of the N-terminal domain of apoE. ApoA-I is colored gold, and apoE is blue. (B) Full-length apoA-I, shown within the elliptical loop formed by the $\Delta 1-43$ structure. Both structures are oriented to place their two largest dimensions in the figure plane and mutually aligned. Helices $A-F$ in the full-length structure are colored as in Fig. 1. Helices in $\Delta 1-43$ are colored to match the sequence limits of the helices in the full-length protein.

secondary structure to the 1-186 fragment in the presence of SDS (32). Stabilization of the four-helix bundle through formation of a hydrophobic core with residues drawn from all four helices makes it likely that any significant truncation of residues in the 1-186 region will disrupt this structure. Helices A-D bury $6,582 \AA^{2}$ of accessible surface area in the interface, with individual helices burying 1,293, 1,486, 1,743, and 2,060 $\AA^{2}$. Helix A contributes nearly $20 \%$ of the buried surface area, unavailable to the $\Delta 1-43$ form. The differences in size and conformation between full-length and $\Delta 1-43$ structures illustrated in Fig. $2 B$ thus are largely due to the essential role of residues 1-43 (helix A) in stabilizing the four-helix bundle. Thus mutants with large deletions, such as $\Delta 1-43$ apoA-I, apoA- $\mathrm{I}_{\text {Seattle, and an 88-98 }}$ deletion mutant (11) are unlikely to form a four-helix bundle. The four-helix parallel bundle adopted by apoA-II is through
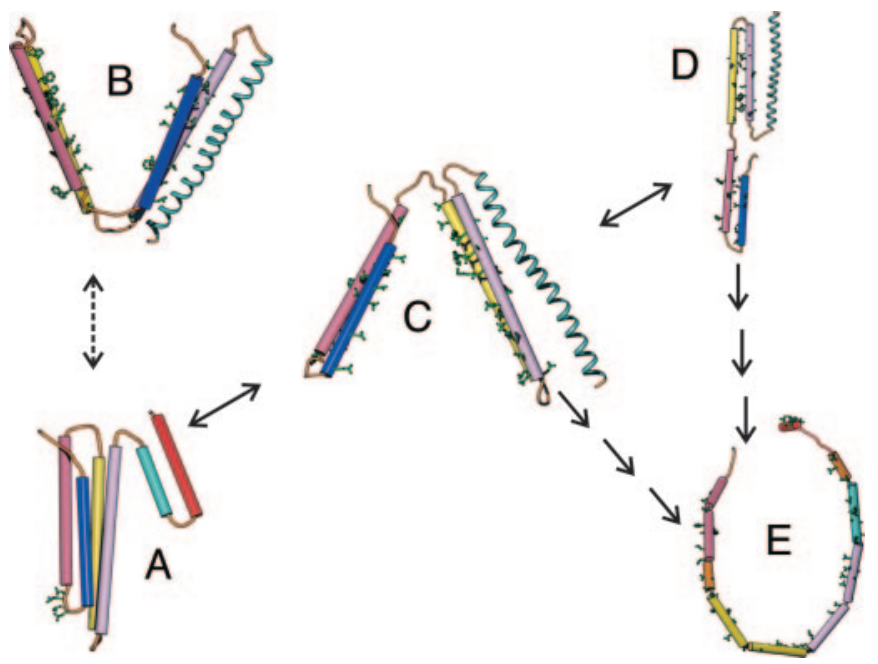

Fig. 3. Model for lipid-assisted conversion. Helices are represented as cylinders and colored as in Fig. 1. Hydrophobic residues are depicted as green sticks. The C-terminal domain is shown as a $\mathrm{C}^{\alpha}$ worm in an arbitrary orientation and position as a single, long helix. $(A)$ Initial lipid-free conformation of apoA-I. Residues contributing to the hydrophobic patch at the $\mathrm{N}$ terminus of helix 1 are shown. ( $B$ and $C$ ) The two geometrically possible open conformations, formed through lipid binding to the bundle. The energetically more likely conformation $(C)$ is indicated by a solid arrow. Hydrophobic side chains that contribute to the four-helix bundle interface are exposed. (D) Rearrangement of a fraction of the open form into a stable helix-hairpin intermediate. Residues forming the new hydrophobic stabilization core are illustrated. $(E)$ Putative conformation in the HDL-bound form, represented by the $\Delta 1-43$ structure. Hydrophobic residues that could potentially form the interaction interface with lipid in HDL are shown.

association of two disulfide bonded dimers (20). Apolipophorins from L. migratoria (19) and M. sexta (33), which form five-helix bundles, also have a similar structural organization to full-length apoA-I. Superimposition over helices 1-4 of the structures of $M$. sexta (97 common $\mathrm{C}^{\alpha}$ atoms) and the L. migratoria $\left(101 \mathrm{C}^{\alpha}\right.$ atoms) yield rms deviations of 3.5 and $4 \AA$, respectively.

Hydrophobic Patch Assists in Lipid-Association. Transfer of cellular cholesterol to apoA-I from peripheral tissues is a two-step process, with the initially bound phospholipids facilitating subsequent efflux of FC (4). Conformational transitions in apoA-I occur in a complex cellular environment that is seldom accessible for direct physical studies. Useful information, however, has been obtained from in vitro model systems, which can, when interpreted with caution, shed light on physiological events. Upon interaction with lipid, the compact lipid-free structure of apoA-I attains an open HDL-associated form, possibly through a helical hairpin intermediate (11). The C-terminal domain, with greatest affinity for lipid in apoA-I, acts as a lipid-sensitive trigger for this transition, with residues 187-243 assuming a helical conformation in the lipid-bound state (34). It has been suggested (26) that disruption of the four-helix bundle occurs subsequently, through a change in conformation of helix A, exposing hydrophobic residues in helix 1 . The crystal structure of apoA-I suggests that lipid binding might assist this step in the process. As depicted in Fig. 3, there are two geometrically possible ways of opening the four-helix bundle $(3 A)$ : one in which the loops ab and cd are used as hinges $(3 B)$ and a second in which the loop bc is used $(3 C)$. Residues in the upper half of the bundle bury $\approx 16 \%$ greater accessible surface area $\left(4,239 \AA^{2}\right)$ than the lower half $\left(3,509 \AA^{2}\right)$, with the two loops ( $a b$ and cd) at the top burying $1,763 \AA^{2}$, nearly twice that of loop bc $\left(915 \AA^{2}\right)$ at the bottom (Fig. 1). Thus, using the bc loop as a hinge is energetically more favorable. An inspection of hydrophobic patches (Fig. 4A) 

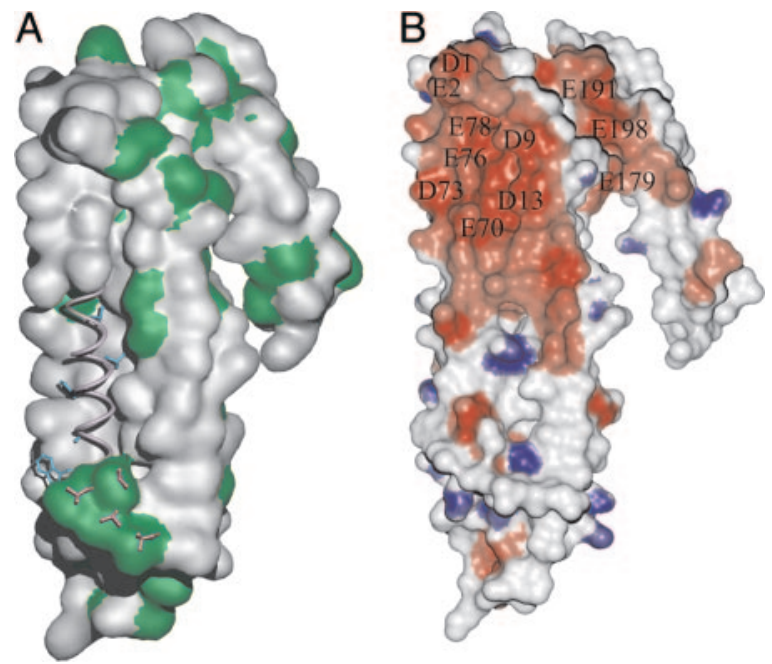

Fig. 4. Surface hydrophobic and electrostatic properties. (A) Solventaccessible surface of apoA-I (gray) with helix 1 residues omitted. Hydrophobic patches are colored green with side chains of leucine quartet colored purple superimposed on the patch at the base of helix $1 . \mathrm{C}^{\alpha}$ worm of helix 1 in light purple and internal hydrophobic residues in cyan are also shown. (B) Positive (blue) and negative (red) electrostatic potential mapped to the molecular surface of apoA-I, oriented to show the largest negative patch. Contours are displayed at \pm 10 e. Positions of side-chain carboxyls are labeled.

on the structure suggests, as the most probable initiation point for the conversion into open structure, the large patch formed by a quartet of leucines, Leu-42 and -44, a part of the ab-loop, and Leu-46 and -47 at the $\mathrm{N}$ terminus of helix 1 (residues 44-65; Fig. $4 A$ ). Helix 1 is the most hydrophobic part of the four-helix bundle, exceeded in hydrophobicity only by C-terminal helix 10 in the whole protein (35). This hydrophobic patch is thus an excellent candidate for initiation of the four-helix bundle disruption through lipid binding, because it is adjacent to Trp-50, Val-53, Phe-57, Leu-60, and Leu-64, which give helix 1 its large hydrophobic moment (Fig. 4A). Because these five residues are part of the hydrophobic core of the four-helix bundle (Fig. 1), binding to the leucine patch would permit lipid molecules access to unravel the bundle. Both energetic feasibility and potential lipid acyl chain access to the hydrophobic core of the four-helix bundle make the open form labeled $3 C$ the more likely intermediate (indicated by a solid arrow). In the absence of significant quantities of lipid, a fraction of $3 C$ could form a transient, fully open form, with subsequent rearrangement yielding a stable helical hairpin $(3 D)$. The structure $3 D$ was obtained through CNS (http://cns.csb.yale.edu) energy minimization of $3 B$, shielding most hydrophobic residues in a new interhelix interface. In the presence of sufficient quantities of lipid to saturate the Cterminal domain, for example in the vicinity of membranes, either $3 B$ or $3 D$ could eventually result in the HDL-bound conformation of apoA-I, represented by the structure of $\Delta 1-43$ apoA-I $(3 E)$. The C-terminal domain, except in $3 A$, is shown in an arbitrary orientation and location, but in a helical conformation similar to that observed in EPR experiments (34). Some of the hydrophobic patches (Fig. $4 A$ ) also might play a role in self-association (16), a property apoA-I shares with apoE (21).

Lipid-mediated unraveling of helical bundles has been proposed for insect apolipoproteins and $\operatorname{apoE}(19,21,33)$. Based on hydrodynamic measurements, a similar model has also been suggested for apoA-I (26). Dimensions of the four-helix bundle $(88 \times 27 \AA)$ and helical hairpin 3D $(163 \times 20 \times 26 \AA)$ are close to that of their hydrodynamic counterparts $(90 \times 29$ and $168 \times$ $24 \AA$, respectively). Our model differs in suggesting a significant role for residues 1-43 in stabilizing helical hairpin intermediates, similar to its role in this structure.

Functional Implications. Analysis of the surface charge distribution on the structure offers clues to interaction of apoA-I with ABCA1 and SR-B1. Two negative patches (Fig. 4B) dominate the electrostatic surface of apoA-I. The largest patch has contributions from residues in the N-terminal domain (Asp-1, -9, and -13 and Glu-2), helix 2 (Asp-73, Glu-76, and Glu-78) whereas Glu-179, -191, and -198 , in helices 7 and 8 contribute to the smaller patch in Fig. $4 B$. The major patch is $\approx 28 \AA$ from the large hydrophobic patch on the C-terminal domain, and the two together might be a potential ABCA1 recognition motif on apoA-I. Recent studies have shown that docking of apoA-I to an extracellular domain of ABCA1 precedes cholesterol acceptance (36). Cross-linking (37) and synthetic peptide (38) studies have indicated that interaction between apoA-I and ABCA1 is not sequence specific. Analysis of helix pairs, such as 1 and 9 in apoA-I, has shown that an important motif for interaction of many lipoproteins with ABCA1 is the C-terminal hydrophobic patch adjacent to a negative charge cluster (39). Although acidic residues, which are part of the proposed ABCA1 interaction motif, are not known with certainty, it is intriguing that the largest negative patch including contributions from Asp-73 and Glu-78, both part of helix 2 , is $\approx 28 \AA$ from the hydrophobic patch on the C-terminal domain; a distance that is close to the $\approx 32 \AA$ estimated to separate similar patches in the helix 1-9 chimera (39). The preponderance of negative charge on the surface results in a predominantly negative potential field surrounding the structure. Electrostatic attraction of apoA-I toward positively charged regions of ABCA1 is hypothesized to be important prelude to their interaction (39). Presence of a negative potential field around apoA-I would assist in the approach and orientation of lipid-free apoA-I for docking with ABCA1.

Offloading of cholesteryl ester to the liver is mediated through interaction of HDL bound apoA-I with SR-B1, whose extracellular domain binds both HDL and low-density lipoproteins (8). SR-B1 can bind both lipid-free and HDL-associated apoA-I at distinct sites (40). Studies using synthetic peptides indicate that the hydrophilic face of apoA-I helices is recognized by SR-B1 (40). Mutations in murine SR-B1, in which Gly or Gln residues were substituted by Arg, indicate that interaction between HDL-bound or free apoA-I and SR-B1 might in part be mediated through positively charged residues in apoA-I (41). The surface of apoA-I (Fig. 4B) shows several potential positively charged docking regions for binding to SR-B1. Identification of these accessible positive charges provides an opportunity for design of site-directed mutagenesis and other experiments to elicit more information about the residues involved in this interaction.

Presence of oxidized lipids in lipoproteins is a known atherogenic risk factor (10), and lipid-free apoA-I is the target for myeloperoxidase-mediated oxidative chlorination and nitration of Tyr-192. Chlorination results in impairment of its ABCA1dependent cholesterol efflux ability and has been hypothesized to be because of greater accessibility of this Tyr residue in lipid-free compared to HDL-bound apoA-I (17). Tyr-192 is part of the d-e loop (Fig. 5, gold) and is the only Tyr in the structure that is completely solvent accessible, providing a rationale for the observed chlorination and nitration pattern in apoA-I.

ApoA-I in nascent discoidal HDL is the most potent apolipoprotein activator of plasma enzyme LCAT, in mature HDL formation (7). Studies on apoA-I mutants, synthetic peptides, and monoclonal antibodies (reviewed in ref. 22) have identified that residues 143-186 of apoA-I interact with LCAT (Fig. 5, magenta). Most of these residues are in helix D (residues 146-187). Residues Val-156, Leu-159, and Pro-165 (Fig. 5, green) form a part of the hydrophobic core and would be 

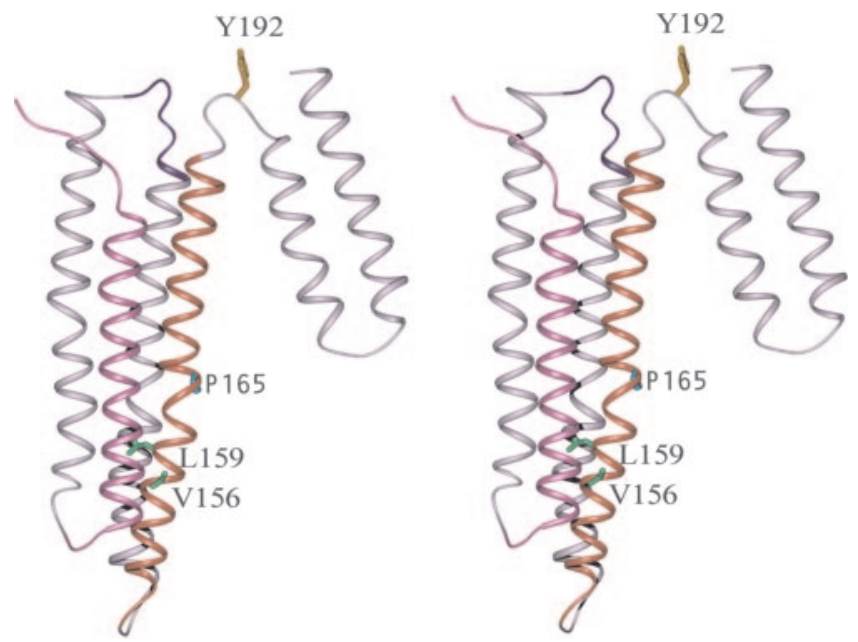

Fig. 5. Functional importance of some residues. Stereoview of $\mathrm{C}^{\alpha}$ worm of lipid-free apoA-I, colored light purple, with N-terminal helix, LCAT interaction region, and residues $98-108$ highlighted in violet, red, and blue, respectively. Side chains of some hydrophobic residues, which interact with LCAT in the lipid-bound form of apoA-I but are part of the hydrophobic core in this structure, are shown in green. Tyr-192, the target for myeloperoxidase, is shown in gold.

unavailable for interaction with LCAT in this conformation. A change in conformation, which occurs upon lipid binding, would expose these residues for interaction with LCAT.

In conclusion, the structure of apoA-I indicates that the $\mathrm{N}$-terminal domain plays a crucial role in maintaining its lipidfree conformation and suggests a possible mechanism for its conversion to a lipid-bound state. It also affords insights into interaction with ligands that are critical to its antiatherogenic properties. The structure also reveals the structural and functional similarity of apoA-I to other apolipoproteins and makes available a structural platform for exploration of molecular mechanisms of its beneficial effects in atherosclerosis and inflammatory diseases.

\section{Materials and Methods}

Crystallization. Delipidated apoA-I was purified from human plasma (42). Crystals were grown by equilibrating $2-\mu 1$ droplets of a $107 \mu \mathrm{M}(3 \mathrm{mg} / \mathrm{ml})$ solution of apoA-I in $20 \mathrm{mM}$ Tris $(\mathrm{pH}$ 7.8) mixed with an equal volume of well solution over wells containing $200 \mathrm{mM}$ Tris (pH 7.8), $100 \mathrm{mM} \mathrm{LiCl,} 50 \mathrm{mM} \mathrm{NaCl}$, $1 \mathrm{mM} \mathrm{NiCl}_{2}, 500 \mu \mathrm{M} \mathrm{Cr}-\mathrm{acac} 3$ (Aldrich), and $11 \%$ polyethylene glycol 4000 , at $20^{\circ} \mathrm{C}$. Appearance of crystals was infrequent, and the structure was determined from four usable crystals, obtained in a large number of trials, which explored salts and coordination compounds of 11 metals as additives. Although our finding that Cr-acac3 was required for growth of suitable crystals was serendipitous, a similar compound has been used to stabilize metmyoglobin in a molten globule conformation (43). The space group was determined to be $\mathrm{P} 4_{1} / \mathrm{P} 4_{3}$, with $a=b=127.8$ and $c=$ $130.1 \AA$ and three apoA-I molecules in the asymmetric unit.

1. Nissen, S. E., Tsunoda, T., Tuzcu, E. M., Schoenhagen, P., Cooper, C. J., Yasin, M., Eaton, G. M., Lauer, M. A., Sheldon, W. S., Grines, C. L., et al. (2003) J. Am. Med. Assoc. 290, 2292-2300.

2. Assmann, G. \& Brewer, H. B., Jr. (1974) Proc. Natl. Acad. Sci. USA 71, 1534-1538.

3. Glomset, J. A. (1968) J. Lipid Res. 9, 155-167.

4. Fielding, P. E., Nagao, K., Hakamata, H., Chimini, G. \& Fielding, C. J. (2000) Biochemistry 39, 14113-14120.

5. Brewer, H. B., Jr. (2004) Arterioscler. Thromb. Vasc. Biol. 24, 387-391.

6. Oram, J. F. (2002) Curr. Opin. Lipidol. 13, 373-381.
Structure Solution and Refinement. The structure was determined by single-wavelength anomalous dispersion phasing (see Table 1, which is published as supporting information on the PNAS web site). Because of the requirement of $\mathrm{Cr}$-acac3 for crystallization, we suspected that $\mathrm{Cr}$ might be present in the lattice, which was confirmed by fluorescence detection of an absorption peak at $2.07 \AA$. All data were measured on flash-frozen crystals. Bijvoet pair data, to $2.8 \AA$, were measured at $2 \AA$, on the SERCAT-ID22 beamline at Advanced Photon Source, using a helium-filled diffracted beam path. At the time the data were measured, this approach was the closest to the $\mathrm{Cr}$ absorption peak permitted by beamline optics. The $\mathrm{f}^{\prime \prime}$ value at $2 \AA$ is $\approx 3.72$ electrons, $\approx 93 \%$ of value at the peak, resulting in an estimated Bijvoet signal of $2.5 \%$ for the seven $\mathrm{Cr}$ ions eventually located. The data used to determine phases were measured from one crystal, in $15^{\circ}$ blocks, by using inverse-beam geometry, processed using DENZO, and were scaled by using an anisotropically parameterized local scaling procedure (44). A data set, edited to remove large differences $[\Delta F>5(R M S \Delta F), 7 \%]$ as well as weak data $(F<$ $2.5 \sigma F, 11 \%)$, which retained $82 \%$ of the measured differences, was used to determine positions of seven $\mathrm{Cr}$ ions in SOLVE (45). Phase refinement with MLPHARE, using the partial structure of the $\mathrm{Cr}$ ions, followed by solvent flattening and noncrystallographic symmetry averaging, using DM, were performed within the CCP4 system (46), improving an initial figure of merit of 0.34 to 0.69 , for all measured data to 3 . An noncrystallographic symmetry-averaged, solvent-flattened electron density map, in space group $\mathrm{P}_{3}$, could be interpreted to locate most of the helical structure in each of the three molecules of apoA-I. The structure was refined in CNS (47) against a maximum likelihood target, by using a complete data set to $2.4 \AA$, derived from merging measurements at $1 \AA$ on three crystals (SERCATID22). Refinement was tightly restrained, using noncrystallographic symmetry restraints, for the protein and $\mathrm{Cr}$-acac3 moieties, in addition to normal geometric restraints. Geometric restraints for the $\mathrm{Cr}$-acac3 moieties were derived from its crystal structure (48). Cross validation was performed throughout the refinement by computing free $R$ values for $5 \%$ of the data, chosen randomly to cover the resolution range. The present $R$ value is 0.231 , with an $R_{\text {free }}$ of 0.245 . Models were built in o (49). Statistics on data measurement, processing, and refinement are presented in Table 1 . The structure, as currently refined, consists of 5,937 protein, 537 solvent, and 154 atoms of seven Cr-acac3 moieties in the asymmetric unit. Strong density implies correct positioning for the only two residues (Gln-41 and Leu-46, in molecule B only), that are in disallowed regions of the Ramachandran map. Figures were composed in RIBBONS (50), GRASP (51), and LIGPLOT (52), and solvent accessibility calculations were carried out by using AREAIMOL (46) with a $1.4-\AA$ probe radius.

We thank K. Judge for technical assistance, V. K. Ganesh for discussions, Z. Jin for help with data measurement, and L. DeLucas for support. This research was supported by National Institutes of Health Grants AI45623, AI51615 (to H.M.K.M.), HL34343 (to G.M.A.), HL64272, and DK46900 (to M.M.H.). The Advanced Photon Source is supported by the U.S. Department of Energy under Contract W-31ch109-Eng-38. G.M.A. is a principal in Bruin Pharma. M.M.H. is president of Chylos, Inc.

\footnotetext{
7. Jonas, A. (2000) Biochim. Biophys. Acta 1529, 245-256.

8. Krieger, M. (1999) Annu. Rev. Biochem. 68, 523-558.

9. Iqbal, J. \& Hussain, M. M. (2005) J. Lipid Res. 46, 1491-1501.

10. Barter, P. J., Nicholls, S., Rye, K. A., Anantharamaiah, G. M., Navab, M. \& Fogelman, A. M. (2004) Circ. Res. 95, 764-772.

11. Brouillette, C. G., Anamtharamaiah, G. M., Engler, J. A. \& Borhani, D. W. (2001) Biochem. Biophys. Acta 1531, 4-46.

12. Marcel, Y. L. \& Kiss, R. S. (2003) Curr. Opin. Lipidol. 14, 151-157.

13. Narayanaswamy, V. \& Ryan, R. O. (2000) Biochem. Biophys. Acta 1483, 15-36.
} 
14. Rothblat, G. H., de la Llera-Moya, M., Atger, V., Kellner-Weibel, G., Williams, D. L. \& Phillips, M. C. (1999) J. Lipid Res. 40, 781-796.

15. Segrest, J. P., Li, L., Anantharamaiah, G. M., Harvey, S. C., Liadaki, K. N. \& Zannis, V. (2000) Curr. Opin. Lipidol. 11, 105-115.

16. Saito, H., Lund-Katz, S. \& Phillips, M. C. (2004) Prog. Lipid Res. 43, 350-380.

17. Shao, B., Bergt, C., Fu, X., Green, P., Voss, J. C., Oda, M. N., Oram, J. F. \& Heinecke, J. W. (2005) J. Biol. Chem. 280, 5983-5993.

18. Borhani, D. W., Rogers, D. P., Engler, J. A. \& Brouillette, C. G. (1997) Proc. Natl. Acad. Sci. USA 94, 12291-12296.

19. Breiter, D. R., Kanost, M. R., Benning, M. M., Wesenberg, G., Law, J. H., Wells, M. A., Rayment, I. \& Holden, H. M. (1991) Biochem. 30, 603-608.

20. Kumar, M. S., Carson, M., Hussain, M. M. \& Murthy, H. M. (2002) Biochemistry 41, 11681-11691.

21. Weisgraber, K. H. (1994) Adv. Protein Chem. 45, 249-302.

22. Sorci-Thomas, M. G. \& Thomas, M. J. (2002) Trends Cardiovasc. Med. 12, 121-128.

23. Li, L., Chen, J., Mishra, V. K., Kurtz, J. A., Cao, D., Klon, A. E., Harvey, S. C., Anantharamaiah, G. M. \& Segrest, J. P. (2004) J. Mol. Biol. 343, 1293-1311.

24. Calabresi, L., Meng, Q. H., Castro, G. R. \& Marcel, Y. L. (1993) Biochemistry 32, 6477-6484.

25. Saito, H., Dhanasekaran, P., Nguyen, D., Deridder, E., Holvoet, P., Lund-Katz, S. \& Phillips, M. C. (2004) J. Biol. Chem. 279, 20974-20981.

26. Rogers, D. P., Roberts, L. M., Lebowitz, J., Datta, G., Anantharamiah, G. M., Engler, J. A. \& Brouillette, C. G. (1998) Biochemistry 37, 11714-11725.

27. Silva, R. A., Hilliard, G. M., Fang, J., Macha, S. \& Davidson, W. S. (2005) Biochemistry 44, 2759-2769.

28. Weers, P. M., Kay, C. M., Oikawa, K., Wientzek, M., Van der Horst, D. J. \& Ryan, R. O. (1994) Biochemistry 33, 3617-3624.

29. Wientzek, M., Kay, C. M., Oikawa, K. \& Ryan, R. O. (1994) J. Biol. Chem. 269, 4605-4612.

30. Gursky, O. \& Atkinson, D. (1996) Proc. Natl. Acad. Sci. USA 93, 2991-2995.

31. Wilson, C., Wardell, M. R., Weisgraber, K. H., Mahley, R. W. \& Agard, D. A. (1991) Science 252, 1817-1822.

32. Okon, M., Frank, P. G., Marcel, Y. L. \& Cushley, R. J. (2001) FEBS Lett. 487, 390-396.

33. Wang, J., Sykes, B. D. \& Ryan, R. O. (2002) Proc. Natl. Acad. Sci. USA 99, 1188-1193.
34. Oda, M. N., Forte, T. M., Ryan, R. O. \& Voss, J. C. (2003) Nat. Struct. Biol. 10, 455-460.

35. Palgunachari, M. N., Mishra, V. K., Lund-Katz, S., Phillips, M. C., Adeyeye, S. O., Alluri, S., Anantharamaiah, G. M. \& Segrest, J. P. (1996) Arterioscler. Thromb. Vasc. Biol. 16, 328-338.

36. Yancey, P. G., Bortnick, A. E., Kellner-Weibel, G., de la Llera-Moya, M., Phillips, M. C. \& Rothblat, G. H. (2003) Arterioscler. Thromb. Vasc. Biol. 23, 712-719.

37. Denis, M., Haidar, B., Marcil, M., Bouvier, M., Krimbou, L. \& Genest, J., Jr. (2004) J. Biol. Chem. 279, 7384-7394.

38. Remaley, A. T., Thomas, F., Stonik, J. A., Demosky, S. J., Bark, S. E., Neufeld, E. B., Bocharov, A. V., Vishnyakova, T. G., Patterson, A. P., Eggerman, T. L., et al. (2003) J. Lipid Res. 44, 828-836.

39. Natarajan, P., Forte, T. M., Chu, B., Phillips, M. C., Oram, J. F. \& Bielicki, J. K. (2004) J. Biol. Chem. 279, 24044-24052.

40. Thuahnai, S. T., Lund-Katz, S., Anantharamaiah, G. M., Williams, D. L. \& Phillips, M. C. (2003) J. Lipid Res. 44, 1132-1142.

41. Gu, X., Lawrence, R. \& Krieger, M. (2000) J. Biol. Chem. 275, 9120-9130.

42. Anantharamaiah, G. M., Hughes, T. A., Iqbal, M., Gawish, A., Neame, P. J., Medley, M. F. \& Segrest, J. P. (1988) J. Lipid Res. 29, 309-318.

43. Blum, O., Haiek, A., Cwikel, D., Dori, Z., Meade, T. J. \& Gray, H. B. (1998) Proc. Natl. Acad. Sci. USA 95, 6659-6662.

44. Hendrickson, W. A. \& Teeter, M. M. (1981) Nature 290, 107-113.

45. Terwilliger, T. C. (2003) Methods Enzymol. 374, 22-37.

46. CCP4 (1993) Acta Crystallogr. D 55, 760-763.

47. Brunger, A. T., Adams, P. D., Clore, G. M., DeLano, W. L., Gros, P., Grosse-Kunstleve, R. W., Jiang, J. S., Kuszewski, J., Nilges, M., Pannu, N. S., et al. (1998) Acta Crystallogr. D 54, 905-921.

48. Diaz-Acosta, I., Baker, J., Cordes, W. \& Pulay, P. (2001) J. Phys. Chem. A 105, $238-244$.

49. Jones, T. A., Zou, J. Y., Cowans, S. W. \& Kjeldgaard, M. (1991) Acto Crystallogr. A 47, 110-119.

50. Carson, M. C. (1997) Methods Enzymol. 277, 493-505.

51. Nicholls, A., Sharp, K. A. \& Honig, B. (1991) Proteins Struct. Funct. Genet. 11, 281-289.

52. Wallace, A. C., Laskowski, R. A. \& Thornton, J. M. (1995) Protein Eng. 8, 127-134. 